\title{
Méthodologie d'analyse et d'optimisation des tolérances dans un contexte de conception intégrée : TOL_ANALYSES
}

\author{
Tolerance Analyses and optimisations \\ methodology in integrated design: \\ TOL_ANALYSES
}

\author{
Abdessalem HASSANI ${ }^{1,2}$, Nizar AIFAOUI ${ }^{1}$, Abdelmajid BENAMARA ${ }^{1}$, \\ Serge SAMPER ${ }^{2}$ \\ ${ }^{1}$ Laboratoire de Génie Mécanique LGM, Ecole Nationale d'Ingénieurs de Monastir, \\ 5, Av. Ibn Eljazzar 5019 Monastir, Tunisie, E-mail : nizar.aifaoui@ipeim.rnu.tn \\ ${ }_{2}$ Laboratoire SYstèmes et Matériaux pour la MEcatronique SYMME, \\ Polytech'Savoie, BP 8043974944 Annecy le Vieux Cedex, France, serge.samper@univ-
} $\underline{\text { savoie.fr }}$

\begin{abstract}
Résumé :
Les pièces d'un lot, théoriquement identiques, ne peuvent réellement pas avoir des dimensions égales. Une cote ne sera réalisable que si l'on tolère un écart par rapport à l'idéal. Ce dernier est déterminé par un couple de grandeurs qui sont soit les bornes d'un intervalle, soit la moyenne et la variance du lot. Le plus souvent, on définit deux états limites maximal et minimal. Ces derniers, appelées tolérances, doivent être déterminés judicieusement. Un tolérancement utilise des indications syntaxiques et sémantiques pour apporter un sens. L'objectif de cet article est de proposer et de valider une méthodologie d'aide au choix et à la vérification des tolérances. Cette méthodologie est basée sur deux méthodes complémentaires : la méthode au pire des cas (intervalles) et la méthode de Monte Carlo (statistique). Une mise en œuvre informatique a montré la faisabilité de l'outil ainsi que l'apport majeur de la méthodologie proposée dans l'aide à la spécification, à l'optimisation et à la vérification des tolérances avec prise en compte des conditions de fabrication.
\end{abstract}

Mots clés : Conception de produit, Tolérancement, Capabilité procédé, Méthodes arithmétiques, Méthodes statistiques, Méthode de Monte Carlo, Spécification, Synthèse, Optimisation.

Abstract: Real parts of a batch are theoretically all identical but physically all different. Their dimensions cannot be the same. A dimension is tolerable if its deviation remains close enough to the ideal value. This deviation is limited by two values, either bounds of an interval or the mean and the variance of a batch. Usually, we define two extreme bonds of dimensions, called tolerances that must be determined wisely. A tolerancing uses syntactic and grammatical information to provide a sense. The aim of this article is to propose and validate a methodology for assistance in the selection and verification of tolerances. This methodology is based on two complementary approaches: the method at worst case and the Monte Carlo method. An implementation computer showed the 
feasibility of the tool as well as the major contribution of the methodology proposed in aid to the specification, optimization and verification of tolerance, taking account of the conditions of manufacture.

Keyword: Product Design, Tolerancing, Capability-process, Arithmetical methods, Statistical methods, Monte Carlo method, Specification, Synthesis, Optimization.

\section{Introduction}

La conception d'un produit industriel est le résultat d'une démarche de conception réfléchie et maîtrisée. Le but est de satisfaire des exigences fonctionnelles provenant du cahier des charges mais aussi des acteurs de la conception. Le coût, le délai et la qualité sont parmi ces exigences. La définition, la réalisation et le contrôle de la géométrie des pièces selon les normes sont des aspects déterminants lors de la satisfaction des exigences de conception.

La fabrication des pièces constituant le produit ne peut être réalisée selon des cotes nominales (idéales), et ce, quelque soit la précision des machines utilisées. Il est par conséquent impératif d'adopter une démarche de spécification et de vérification des tolérances lors de la définition de la géométrie des pièces composant l'assemblage. La démarche de tolérancement consiste à définir des états limites à chaque cote nominale associée à la géométrie. Plus les tolérances sont larges, plus les coûts d'assemblage sont élevés. Pour assurer ce compromis et permettre la minimisation des coûts de non qualité et de non-conformité du produit, il est impératif de mettre en place une stratégie d'aide à la spécification judicieuse des tolérances. Cette spécification constitue un vecteur de communication entre la conception et la fabrication.

Un système performant en CFAO se doit également de proposer au concepteur une aide au tolérancement fonctionnel respectant les normes et les standards internationaux. Cette aide peut se manifester par des simulations numériques du tolérancement des assemblages lors de la conception. Cela permettra par exemple de prévoir dès la conception les risques de disfonctionnement des assemblages. Cela conduira par exemple à synthétiser des valeurs de tolérances sur la base d'un certain pourcentage de rebut de pièces (taux de nonconformité).

L'objectif de cet article est de proposer une approche originale de spécification et de vérification des tolérances en se basant à la fois sur des méthodes arithmétiques et statistiques. Un état de l'art sur les principales méthodes d'analyse et de synthèse (optimisation) des tolérances ainsi que les outils de Tolérancement Assisté par Ordinateur (TAO) permettra de mettre en évidence le problème de recherche ainsi que les grandes lignes de l'approche proposée. Ensuite, la méthode d'aide à l'analyse et à la synthèse des tolérances est détaillée. Le maquettage de notre méthode a été développée dans l'environnement de programmation Matlab. Un exemple de validations permettra enfin de souligner la mise en œuvre de la méthode dans un processus de choix et de vérification des tolérances dans un contexte de conception intégré. 


\section{Etat de l'art}

Selon la norme ISO 2768, la tolérance ou l'intervalle de tolérance est l'étendue de la variation entre la forme nominale et la dimension. Ainsi, une tolérance est une spécification exprimée en termes d'écart algébrique admissible entre la grandeur réelle et la grandeur théorique [1]. Elle présente la différence entre les valeurs maximale et minimale de la variation d'une valeur donnée. C'est la variation permise (tolérée, admissible) de la cote réelle de la pièce.

D'après les normes ISO-8015 et NF E 04-561, il existe deux types de tolérances ; les tolérances dimensionnelles et les tolérances géométriques. Dans la figure 1, les tolérances dimensionnelles comportent les tolérances linéaires et angulaires. Tandis que les tolérances géométriques regroupent les tolérances d'orientation, de position et de battement [1].

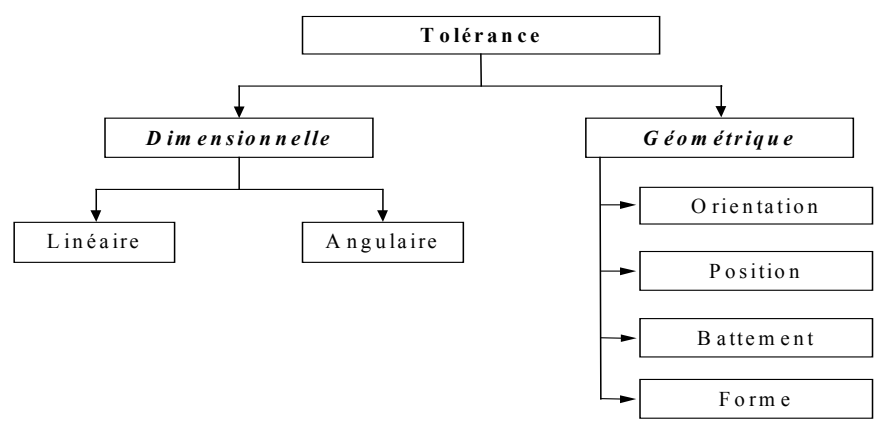

Figure 1 : Typologie des tolérances.

La spécification des tolérances est basée sur un certain nombre de principes et d'exigences. Par exemple, le principe de l'indépendance [1,2] défini par les normes NF E 04-561 1991 et ISO 8015 décrit que chaque exigence doit être respectée en elle-même sauf indication particulière spécifiée. D'autre part, l'exigence de l'enveloppe, définie par la norme ISO 8015 [1,2], délimite la forme de la pièce. Ainsi, l'enveloppe de la forme parfaite au maximum de matière ne doit pas être dépassée. D'autres relations sont également définies sous les termes d'exigence de maximum de matière et d'exigence de minimum de matière. À ces effets, par la suite chaque spécification sera traitée indépendamment des autres.

Dans les paragraphes suivants, une revue sur les principales méthodes d'analyse et de d'optimisation des tolérances est introduite.

La spécification et la vérification des tolérances consistent à effectuer écriture puis une analyse et/ou une optimisation des tolérances. L'analyse des tolérances consiste à vérifier à postériorité la réalisation des conditions fonctionnelles préalablement spécifiées à partir du tolérancement instancié de toutes les pièces du mécanisme [1,2,3]. Tandis que, la synthèse (optimisation des valeurs) des tolérances consiste, à partir des spécifications exprimant les conditions fonctionnelles entre pièces, à générer un 
tolérancement des différentes pièces du mécanisme étudié respectant ces conditions $[4,5$, $6,7]$. Ces deux approches permettent donc la vérification de la conformité d'un assemblage en prenant en compte la variation de différents composants en respectant des conditions fonctionnelles.

\section{II.1 Synthèse sur les principales méthodes}

Dans $[3,4,5,6,7,8,9,10,11]$, différentes méthodes d'analyse et de synthèse des tolérances ont été recensés. Elles se classifient en deux grandes familles : les méthodes arithmétiques et les méthodes statistiques.

D'une façon générale, la formulation mathématique d'un problème d'analyse ou de synthèse des tolérances assure une relation entre les variables d'entrée $\mathrm{X}$ et les dimensions à contrôler (cote condition) $\mathrm{Y}:[4,5]: \mathrm{Y}=\mathrm{f}(\mathrm{X} 1, \mathrm{X} 2, \ldots, \mathrm{Xn})$.

\section{a- Les méthodes arithmétiques}

Les méthodes arithmétiques supposent que les tolérances sont à leurs limites extrêmes tout en garantissant l'assemblabilité de toutes les pièces. Ce qui garantit l'interchangeabilité totale des composants de l'assemblage. Ce type de tolérancement est appelé aussi tolérancement « au pire des cas ». Parmi les méthodes arithmétiques, il y a :

- La méthode des chaînes des cotes $[6,7]$,

- La méthode des domaines de jeux des écarts $[6,7]$.

- La méthode des TMAP [12]

\section{b- Les méthodes statistiques}

Aujourd'hui, de nombreuses industries s'intéressent de très près au tolérancement statistique [7] car il est plus proche du réel que le tolérancement au pire des cas :

- Le principal argument réside dans la réduction des coûts de production. Le tolérancement statistique adopte une stratégie différente et surtout moins coûteuse : augmenter les intervalles de tolérance sur les spécifications en acceptant de perdre l'avantage de l'interchangeabilité totale en quantifiant le pourcentage d'échec d'assemblage appelé aussi Taux de Non-Conformité (TNC) à définir judicieusement.

- Dans certaines industries telle que l'industrie électronique, le tolérancement statistique s'impose car le taux de miniaturisation dépasse largement les améliorations des procédés de fabrication.

- Le tolérancement statistique prend en compte les conditions de production sous plusieurs formes telles que la capabilité machine ou encore le risque moyen. Ce qui répond aux principes de base de l'ingénierie simultanée et concourante.

Grand nombre des travaux de recherche actuels s'orientent vers les méthodes statistiques du fait de la flexibilité qu'elles peuvent donner au concepteur lors de l'expression des contraintes dimensionnelles et géométriques conditionnant le bon fonctionnement d'un mécanisme au juste coût. Parmi les méthodes statistiques $[5,6,7,8,9,10,11]$, il y a la méthode :

- de la racine de la somme carrée, $[5,6,7]$ 
- de Croft, $[5,6,7]$

- de la série de Taylor, $[5,6,7]$

- inertielle, $[8,9,10,11,13]$

- de Taguchi, $[5,8,9,11,13]$

- de Monte Carlo [5, 6, 7, 14, 15, 16]

\section{c- Tableau récapitulatif}

Dans [5], les méthodes arithmétiques et statistiques sont largement détaillées. Dans le tableau suivant, les principaux avantages et inconvénients de ces différentes méthodes sont exposés.

\begin{tabular}{|c|c|c|c|c|}
\hline \multicolumn{2}{|c|}{ Méthodes } & Principe & Avantages & Inconvénients \\
\hline \multirow{2}{*}{ |ֶ: } & Chaîne de cotes & $\begin{array}{l}\text { Calcule la cote condition à } \\
\text { partir de la chaîne de cotes. }\end{array}$ & $\begin{array}{l}\text { Mise en œuvre simple. } \\
\text { Assure l'interchangeabilité } \\
\text { totale des pièces et } \\
\text { assemblages. }\end{array}$ & $\begin{array}{l}\text { Limitée les tolérances } \\
\text { dimensionnelles. } \\
\text { Appliquée aux solides } \\
\text { rigides } \\
\text { Gourmande en temps de } \\
\text { calcul. }\end{array}$ \\
\hline & $\begin{array}{l}\text { Domaine des } \\
\text { jeux et écarts }\end{array}$ & $\begin{array}{l}\text { Opère une linéarisation des } \\
\text { déplacements infinitésimaus } \\
\text { d'un solide. } \\
\text { Enonce les équations } \\
\text { fonctionnelles } \\
\text { Teste l'assemblabilité d'un } \\
\text { mécanisme. }\end{array}$ & $\begin{array}{l}\text { Modélise graphiquement les } \\
\text { zones de tolérances. } \\
\text { Prend en compte les jeux } \\
\text { dimensionnels et angulaires. }\end{array}$ & $\begin{array}{l}\text { Appliquée aux solides } \\
\text { rigides. } \\
\text { Gourmande en temps de } \\
\text { calcul. }\end{array}$ \\
\hline \multirow{7}{*}{. } & $\begin{array}{l}\text { Racine de la } \\
\text { somme carrée }\end{array}$ & $\begin{array}{l}\text { Calcule la cote condition à } \\
\text { partir de la série de Taylor. }\end{array}$ & Simplicité de mise en œuvre. & $\begin{array}{l}\text { Utilisée pour des fonctions } \\
\text { linéaires de la cote } \\
\text { condition. }\end{array}$ \\
\hline & Croft & $\begin{array}{l}\text { Approxime une distribution } \\
\text { tronquée par une } \\
\text { distribution rectangulaire. }\end{array}$ & $\begin{array}{l}\text { Meilleure que la méthode de } \\
\text { la racine carrée pour un } \\
\text { nombre restreint de } \\
\text { paramètres. }\end{array}$ & $\begin{array}{l}\text { Probabilité d'apparition des } \\
\text { valeurs extrêmes est la } \\
\text { même que toutes les autres } \\
\text { valeurs. }\end{array}$ \\
\hline & Série de Taylor & $\begin{array}{l}\text { Résoud analytiquement les } \\
\text { expressions des chaînes de } \\
\text { cotes. }\end{array}$ & $\begin{array}{l}\text { Calcul de l'expression de la } \\
\text { chaîne de cotes linéaires et } \\
\text { non linéaire. }\end{array}$ & $\begin{array}{l}\text { Complexité des dérivées } \\
\text { partielles pour le calcul des } \\
\text { chaînes non linéaires. }\end{array}$ \\
\hline & Inertielle & $\begin{array}{l}\text { Utilise la fonction coût de } \\
\text { Taguchi. } \\
\text { Minimise les inerties. }\end{array}$ & $\begin{array}{l}\text { distributions aléatoires des } \\
\text { écarts. } \\
\text { Meilleur compromis coût de } \\
\text { production/qualité. }\end{array}$ & $\begin{array}{l}\text { Acceptabilité limitée par la } \\
\text { dépendance de l'élément à } \\
\text { la population étudiée. }\end{array}$ \\
\hline & Taguchi & $\begin{array}{l}\text { Utilise la fonction coût de } \\
\text { Taguchi } \\
\text { Assure une distribution en } 3 \\
\text { temps. }\end{array}$ & $\begin{array}{l}\text { - Simplicité } \\
\text { - Application pour les } \\
\text { distributions normales. }\end{array}$ & $\begin{array}{l}\text { Difficulté d'application } \\
\text { pour les distributions non } \\
\text { normales. } \\
\text { Résultats sur les moments. }\end{array}$ \\
\hline & Monte Carlo & $\begin{array}{l}\text { Echantillonne aléatoirement } \\
\text { les distributions } \\
\text { Simule numériquement. }\end{array}$ & $\begin{array}{l}\text { Simplicité de mise en œuvre } \\
\text { Très utilisée. } \\
\text { Précise pour N importante } \\
\text { Application avec une loi } \\
\text { quelconque. }\end{array}$ & $\begin{array}{l}\text { Nombre d'itérations } \mathrm{N} \\
\text { important. } \\
\text { Gourmande en temps de } \\
\text { calcul. }\end{array}$ \\
\hline & \begin{tabular}{|c|} 
Quadratique ou \\
approximation \\
par intégration \\
numérique
\end{tabular} & $\begin{array}{l}\text { - Détermine les dérivées } \\
\text { partielles de la chaîne de } \\
\text { cotes. }\end{array}$ & $\begin{array}{l}\text { Etude des cas non linéaires et } \\
\text { pour le calcul des dérivées } \\
\text { partielles. }\end{array}$ & $\begin{array}{l}\text { Gourmande en temps de } \\
\text { calcul. }\end{array}$ \\
\hline
\end{tabular}

Tableau 1. Etude comparative des différentes méthodes

Parallèlement à ces travaux de recherche, diverses contributions ont été recensées dans le domaine des systèmes d'assistance au tolérancement. Ces système sont dénommés: 
Tolérancement Assisté par Ordinateur (TAO) ou encore Computer Aided Tolerancing (CAT). Depuis des années, ces outils souffrent du manque d'intégration aux outils de CFAO. Aujourd'hui, ce constat n'est plus vrai, car ces outils de TAO commencent s'intégrer aux outils de CFAO [16, 17, 18] et à aborder différents problèmes de tolérancement tel que la spécification et la vérification. Quelques outils de TAO sont recensés dans le paragraphe suivant.

\section{Il.2 Outils commercialisés de TAO}

L'émergence des ingénieries de conception tel que l'ingénierie simultanée, l'ingénierie concourante et l'ingénierie collaborative a favorisée l'intégration des outils de TAO dans les outils de CFAO. Au début, ces outils traitaient des problèmes simples de type chaînes de cotes unidirectionnelles [17]. Ensuite, ils se sont focalisés sur des problèmes plus complexes de synthèse et d'analyse des tolérances. Le tableau non exhaustif suivant regroupe quelques outils de TAO et précise les avantages et les inconvénients de chacun.

\begin{tabular}{|l|l|l|l|}
\hline \multicolumn{1}{|c|}{ Outils } & \multicolumn{1}{c|}{ Principe } & \multicolumn{1}{c|}{ Avantages } & \multicolumn{1}{c|}{ Inconvénients } \\
\hline $\begin{array}{l}\text { TOLTECH } \\
\text { (TOLerance } \\
\text { TECHnology) }\end{array}$ & $\begin{array}{l}\text { Calcul de tolérances des } \\
\text { chaînes de cotes } \\
\text { unidimensionnelles } \\
\text { Optimiser les tolérances }\end{array}$ & $\begin{array}{l}\text { Minimiser le coût de } \\
\text { fabrication }\end{array}$ & $\begin{array}{l}\text { Coût élevé. } \\
\text { Non disponible } \\
\text { commercialement. }\end{array}$ \\
\hline $\begin{array}{l}\text { CATIA } \\
\text { Tolerancing }\end{array}$ & $\begin{array}{l}\text { Spécifier et valider les } \\
\text { tolérances }\end{array}$ & $\begin{array}{l}\text { Intégration à l'outil de CFAO } \\
\text { CATIA }\end{array}$ & $\begin{array}{l}\text { Tolérances sous forme } \\
\text { d'annotations au niveau } \\
\text { maquette de CFAO. }\end{array}$ \\
\hline $\begin{array}{l}\text { Valysis du module } \\
\text { ValysisDesign }\end{array}$ & $\begin{array}{l}\text { Spécifier et vérifier les } \\
\text { tolérances. }\end{array}$ & $\begin{array}{l}\text { Spécification et vérification } \\
\text { des tolérances géométriques } \\
\text { et dimensionnelles ainsi } \\
\text { qu'une analyse des tolérances } \\
\text { dimensionnelles. }\end{array}$ & $\begin{array}{l}\text { Coût élevé et non } \\
\text { disponibilité } \\
\text { commercialement. }\end{array}$ \\
\hline $\begin{array}{l}\text { TASYS } \\
\text { SolidWorks }\end{array}$ & $\begin{array}{l}\text { delérancement des } \\
\text { composants sur } \\
\text { l'assemblage global }\end{array}$ & Analyser les tolérances & $\begin{array}{l}\text { Tolérances sous forme } \\
\text { d'annotations au niveau } \\
\text { maquette de CAO. }\end{array}$ \\
\hline
\end{tabular}

Tableau 2 . Caractéristiques de quelques outils de TAO

\section{Position du problème de recherche}

A la lumière de cet état de l'art, le concept de tolérancement peut être considéré comme un vecteur de communication entre la conception et la fabrication. Plusieurs travaux de recherche se sont focalisés sur la prise en compte de cette étape par la mise en œuvre d'outils d'assistance à la spécification et à la vérification des tolérances de produits mécaniques. Des méthodes arithmétiques et statistiques ont été développées pour assister la mise en œuvre des outils de TAO. Le présent travail propose une méthodologie de spécification et de vérification des tolérances basée sur deux méthodes complémentaires à savoir: la méthode au pire des cas qui est une méthode arithmétique et la méthode de Monte Carlo qui est une méthode statistique. Cette méthodologie permettra au concepteur, en fonction de la nature du problème, de vérifier un tolérancement déjà spécifié en respectant une cote condition. Elle permet également de spécifier des tolérances dans le respect d'une cote condition et ce par application de l'une des deux 
méthodes. Dans tout état de cause, le concepteur est assisté dans les choix à faire (figure 2). Nous partons du fait que dans la pratique, les valeurs des tolérances allouées par les ingénieurs d'étude correspondent rarement à des solutions optimales en raison du peu de données dont ils disposent $[3,18,19,20]$. Cela est appuyé par des déclarations de responsables au sein d'entreprises. Afin de montrer l'apport de la proposition dans un contexte de conception intégrée, un exemple de spécification de tolérances lors de la conception d'un produit mécanique sera développé.

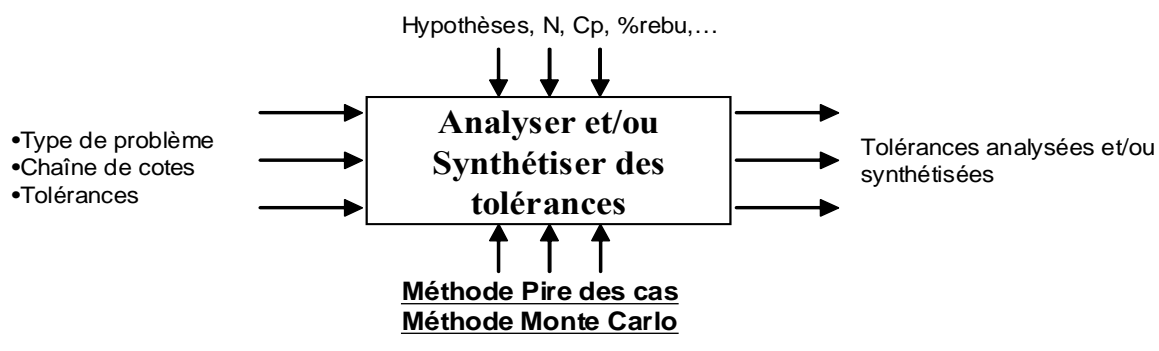

Figure 2 : Modèle SADT de résolution d'un problème de tolérancement.

\section{Modèle proposé}

\section{IV.1. Hypothèses de modélisation}

La méthodologie proposée est basée sur les hypothèses suivantes :

- Seul le tolérancement dimensionnel est considéré.

- Seules les chaînes de cotes linéaires sont considérées

- Les pièces étudiées sont considérées comme des solides rigides. Les déformations dues aux conditions de montage et de fonctionnement ne sont pas prises en considération.

- Le principe de l'indépendance entre les tolérances est retenu.

Un mécanisme est constitué de différentes pièces. Pour que ce dernier soit fonctionnel des conditions doivent être assurées tel que : jeu, dépassement ou retrait. Ces conditions caractérisent la cote condition. Sa variation est liée aux cotes des pièces participantes dans la chaîne. Une chaîne de cotes peut être de deux types (figure 3) :

- Si la cote condition a le même sens que les autres cotes de la chaîne, elle est définie par la somme des cotes participantes à l'assemblage.

- Si la chaîne de cotes dispose de cotes ayant un sens opposé à la cote condition, elle sera calculée par une combinaison de sommes et de soustractions des cotes participantes. 


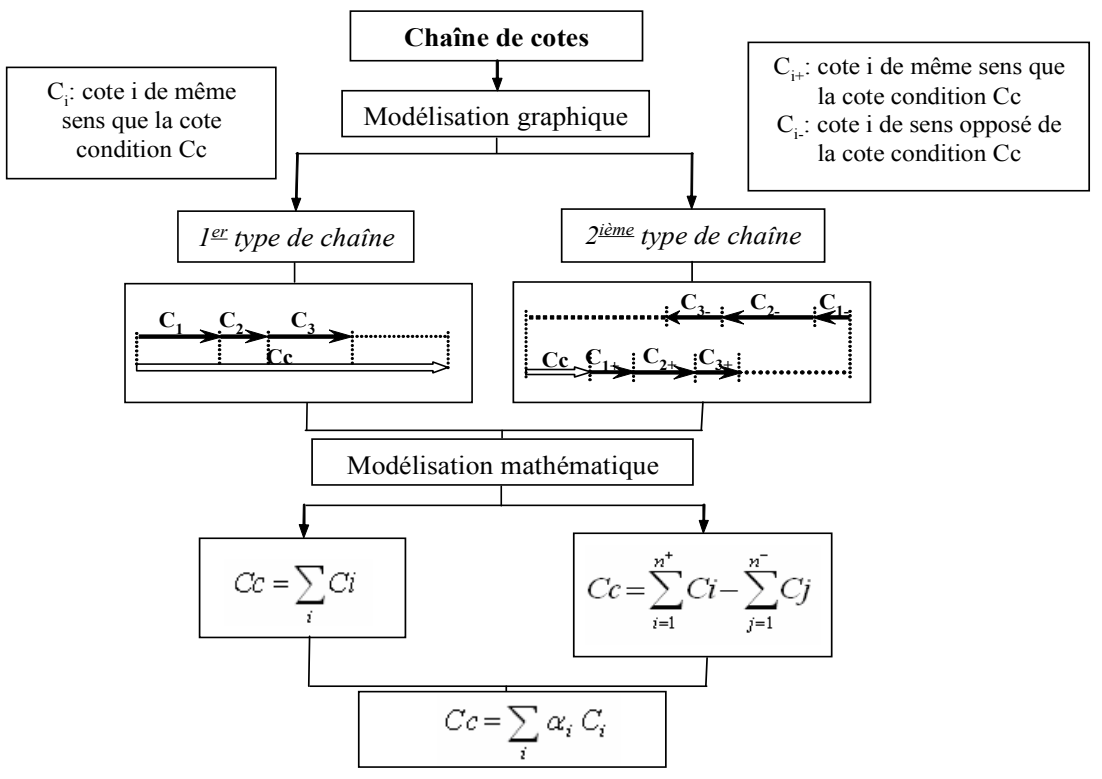

Figure 3 : Modélisation des chaînes de cotes

Avec :

- $n^{+}$: nombre de pièces selon la cote condition Cc

- $\quad n^{-}$: nombre de pièces selon le sens inverse de la cote condition $\mathrm{Cc}$

- $\alpha=1$ pour les cotes de même sens que la cote condition, $\alpha=-1$ pour les cotes de sens opposées que la cote condition.

D'autre part, les tolérances peuvent avoir une répartition [3, 19], (figure 4) :

- Uniforme : les intervalles de tolérances des cotes participantes à la chaîne sont égaux.

- Non uniforme : les intervalles des tolérances des cotes participantes à chaîne diffèrent d'une cote à un autre.

Par ailleurs, chaque tolérance associée à une cote peut être (figure 5) :

- Symétrique : l'écart supérieur de la tolérance est égal à l'écart inférieur en valeur algébrique.

- Dissymétrique : les valeurs attribuées aux écarts supérieur et inférieur sont différentes algébriquement. 


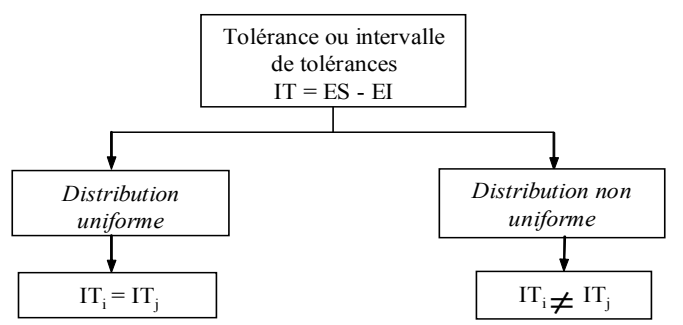

Figure 4 : Répartition des tolérances.

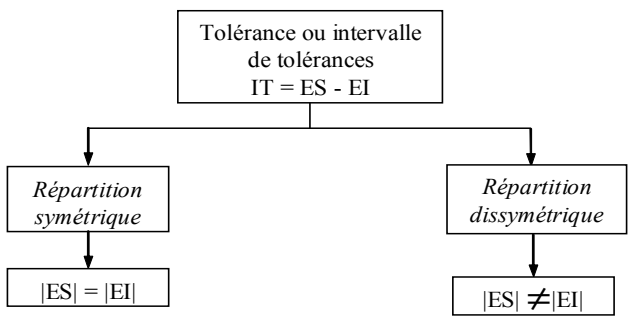

Figure 5 : Répartition des écarts.

\section{IV.2. Algorithme général}

La méthodologie proposée permet au concepteur de mener deux types de calcul: un calcul d'analyse de tolérances et/ou un calcul de synthèse de tolérances sur la base d'une chaîne de cotes prédéfinie et dans le respect d'une cote condition. Pour mener à bien ce calcul, un choix entre deux méthodes est envisageable. La Méthode au Pire des Cas (MPC) et la Méthode de Monte Carlo (MMC) (figure 6).

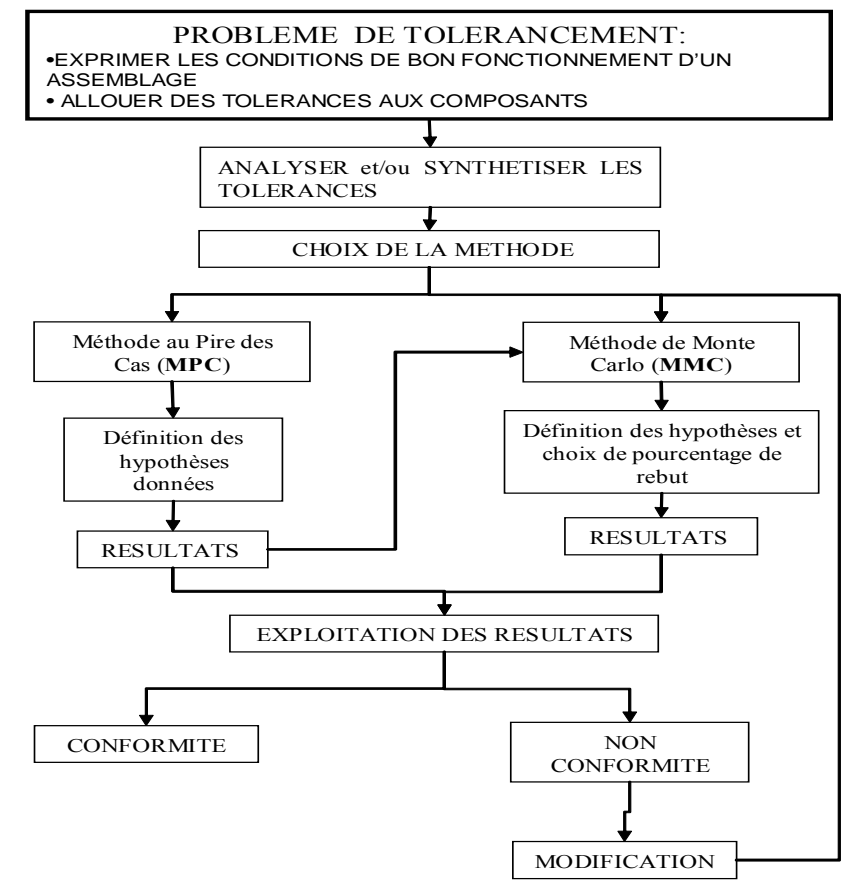

Figure 6: Algorithme général

La mise en œuvre de la méthode (MPC) permet d'analyser un tolérancement prédéfini ou de synthétiser une cote condition à partir de la chaîne de cotes préétablie. L'application de la méthode (MMC) a pour objectif d'élargir des tolérances strictes ou de rétrécir des tolérances larges dans le respect d'un \% de rebut choisi par le groupe de conception. 
La figure 7 représente un exemple de mise en équations d'une chaîne de cotes relative à un assemblage de deux pièces prismatiques.

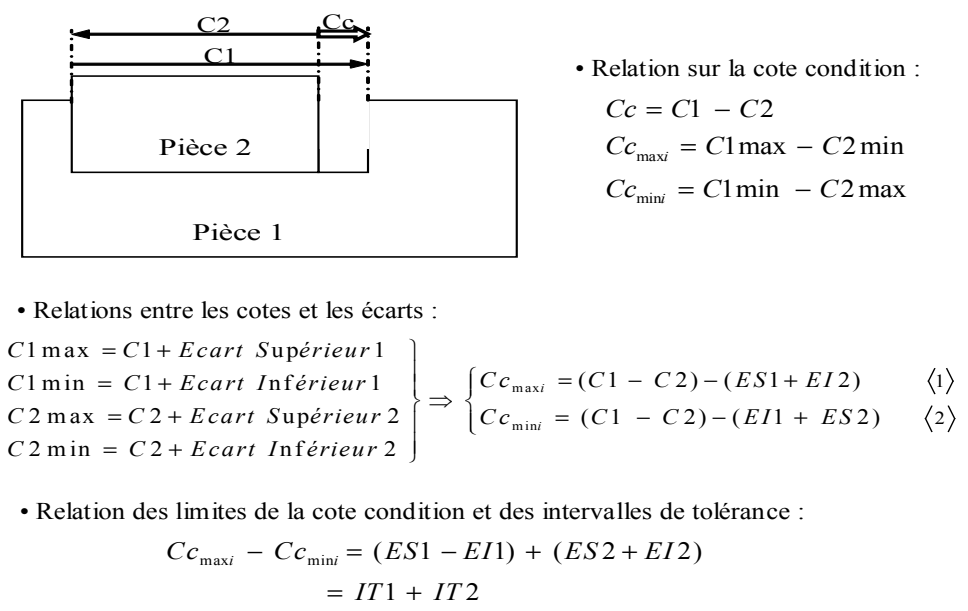

Figure 7: Mise en équations d'une chaîne de cote

Afin d'identifier les principales étapes de mise en œuvre de la méthodologie proposée, les deux méthodes MPC et MMC seront détaillées dans les paragraphes suivants.

\section{IV.1.1. Méthode au Pire de Cas (MPC)}

La Méthode au Pire des Cas (MPC) est une méthode arithmétique permettant l'étude des tolérances des pièces mécaniques dans leurs limites. Elle est basée sur l'interchangeabilité totale des pièces. L'étude des tolérances consiste soit à synthétiser des tolérances dans le respect de la cote condition imposée par la chaîne de cotes, soit d'analyser un tolérancement préétabli. Ce dernier type nécessite de modifier plusieurs fois les tolérances afin d'aboutir aux limites acceptables par la chaîne de cotes de l'assemblage. La MPC permet de résoudre des problèmes dont le nombre d'inconnues est égale à deux. Cette méthode est indifférente vis à vis de la nature des cotes (uniforme ou non, symétrique ou non). Dans la figure suivante, un exemple d'assemblage de deux pièces illustre le principe de la méthode. 

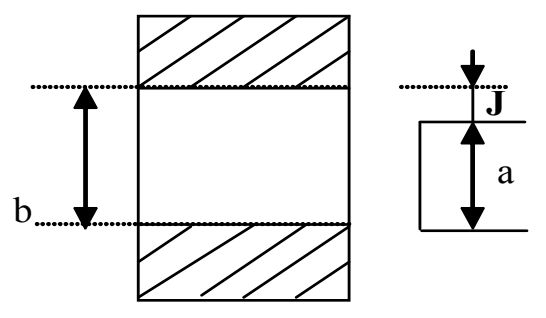

Avec:

Jmax $=$ contenant $\max -$ contenu $\min =$ bmax - amin

Jmin = contenant $\min -$ contenu $\max =$ bmin - amax

Jmax - Jmin $=$ ITa + ITb

Figure 8 : Assemblage de deux pièces

\section{$1^{e r}$ cas : Synthèse de la cote condition}

La synthèse de la cote condition consiste à introduire les cotes avec leurs écarts tout en respectant celles qui sont dans le même sens ou de sens contraire que la cote condition. Il s'agit ensuite de calculer la cote condition avec ses écarts supérieur et inférieur (figure 9-A).

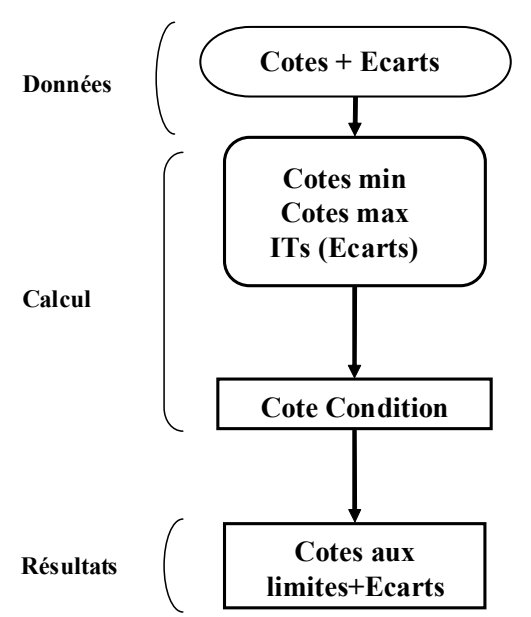

A-Synthèse de la Cote Condition

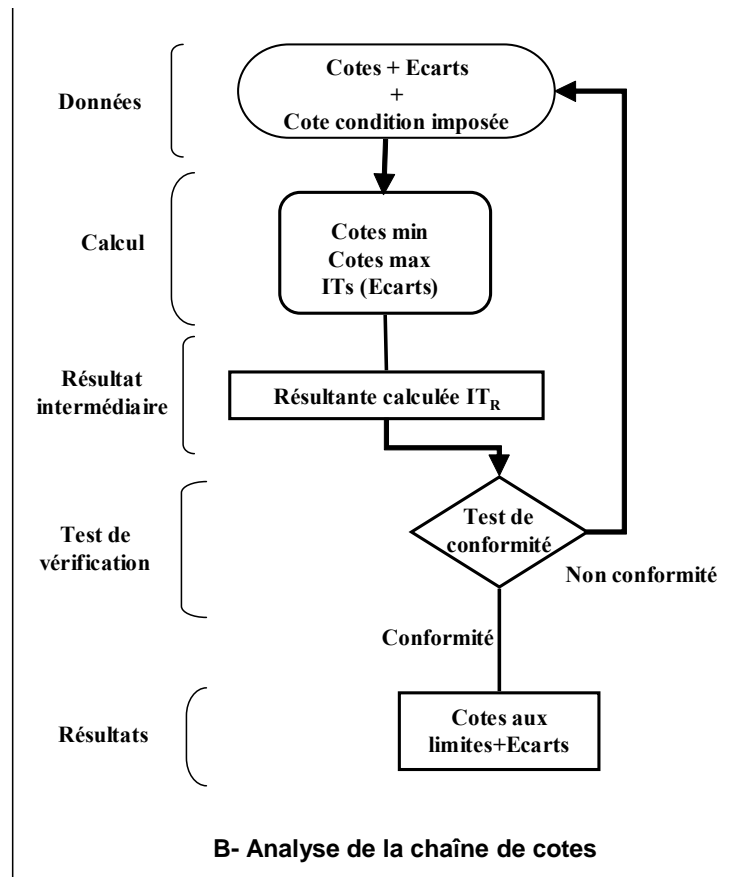

Figure 9: Organigramme d'analyse et de synthèse des tolérances par la MPC

\section{$2^{\text {ème }}$ cas : Analyse de la chaîne de cotes}

L'analyse de la chaîne de cotes consiste à partir d'une chaîne prétablie où la cote condition est imposée. Ensuite, il s'agit de calculer la nouvelle cote condition et de voir 
sa conformité avec celle imposée. Un test de conformité permet de savoir que dans le cas de non-conformité, le concepteur est amené à corriger les tolérances associées aux cotes participantes afin d'assurer la conformité. Les formules suivantes illustrent le principe de cette étape d'analyse. L'organigramme de la figure 9-B illustre les principales étapes de la démarche de vérification de la chaîne de cotes.

$$
\begin{aligned}
\text { cote condition minimale } & \leq \text { cote imposée } \leq \text { cote condition maximale } \\
C c \min & \leq \text { cote imposée } \leq C c \max
\end{aligned}
$$

Pour mieux prévoir les résultats de la fabrication, divers travaux de recherche s'orientent vers l'application de la Méthode de Monte Carlo (MMC) [4, 5, 6, 7, 8, 9]. Cette méthode sera détaillée dans la section suivante.

\section{IV.1.2. Méthode de Monte Carlo (MMC)}

La méthode MMC se base sur le constat suivant: Généralement, la fabrication d'un lot de pièces a une répartition en forme de cloche dont la disposition et la répartition se fait autour d'une moyenne cible (cote nominale) et présente un indicateur de réglage du procédé (machine). Celui-ci est centré autour de la moyenne. Si la production n'a pas la forme d'une cloche centrée, c'est qu'il y a un problème au niveau du procédé d'obtention des pièces (décentrage, troncature,... ) (figure 11). Ce sont des indicateurs de non-conformité du produit qui nous conduisent à l'étude de la capabilité du procédé [14, $15,21]$. La MMC utilise des générateurs pseudo-aléatoires de nombres correspondant à différents types de distributions (Student, gaussienne, etc.) et permet ainsi de fournir des résultats plus proches de la réalité que les méthodes de calcul standards. La figure suivante représente la démarche générale d'application de cette méthode.

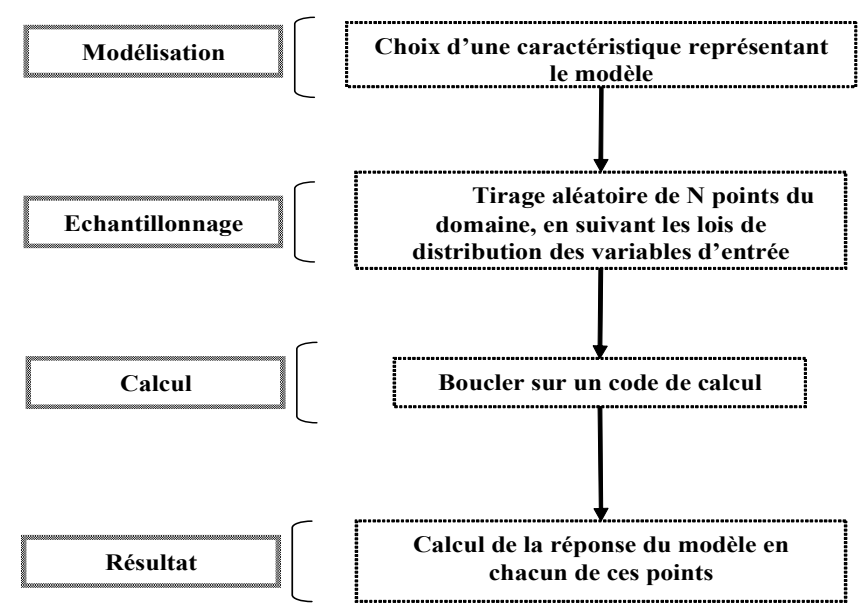

Figure 10: Démarche générale de la méthode MMC 
L'application de la méthode MMC débute par l'identification de la chaîne de cotes et les éléments contributeurs à cette chaîne. L'échantillonnage consiste à effectuer un tirage aléatoire sur chaque caractéristique selon la loi normale. Cette loi a été choisie car elle est représentative de ce qui se produit réellement en production. Cette étape est suivie par la phase de calcul et de simulation des valeurs des itérations.

La simulation à l'aide de la méthode MMC nécessite de connaître la valeur autour de laquelle sera faite la génération des valeurs aléatoires. Ainsi, le problème de répartition des écarts trouve son importance pour identifier la valeur moyenne autour de laquelle sera faite la simulation. Pour la répartition symétrique, la valeur moyenne de chaque caractéristique générée coïncide avec la valeur nominale. Par contre, pour la répartition dissymétrique, la valeur moyenne est déterminée par une opération de centrage de la répartition décentrée. Cela est assuré par l'algorithme suivant.

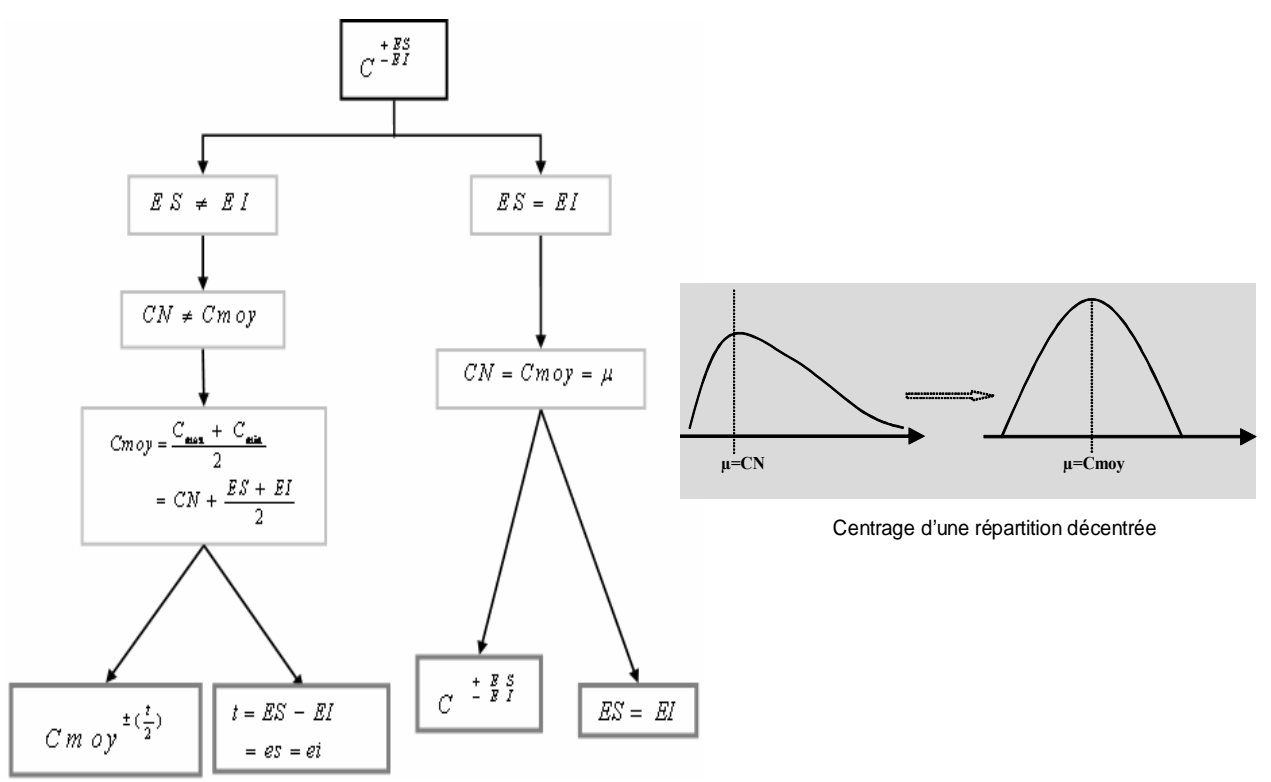

Figure 11: Algorithme de centrage des valeurs des cotes

La loi normale représentant la dispersion de $\mathrm{N}$ pièces autour de la valeur moyenne est caractérisée par une moyenne et un écart type $(\sigma)$. Ce dernier est déterminé à partir de l'indice de capabilité. L'indice de capabilité $(\mathrm{Cp})[3,15,21,22]$ est une mesure établissant un lien entre la dispersion réelle d'une machine (ou procédé) et la dispersion demandée. Il est donné par le rapport entre l'intervalle de tolérance et la dispersion du procédé.

$$
C p=\frac{\text { Intervalle de tolérance }}{\text { Dispersion } \quad d u \text { procédé }}=\frac{I T}{D}=\frac{I T}{6 \sigma}
$$


Ainsi, l'écart type est donné par :

$$
\sigma=\frac{I T}{6 C p}
$$

D'après [3, 21, 22], le processus sera capable et la fabrication des pièces sera bonne pour un indice de capabilité est égal à l'unité $(\mathrm{Cp}=1)$. Donc :

$$
\sigma=\frac{I T}{6}
$$

Cette relation permet de lier l'écart type $(\sigma)$ à l'intervalle de tolérance (IT). La figure 12 montre l'algorithme d'application de la MMC. Cet algorithme permet, à partir des données du problème de vérifier un tolérancement déjà spécifié par comparaison du pourcentage de rebut calculé au pourcentage de rebut imposé. Il permet également, sur la base de cette comparaison de spécifier à nouveau le tolérancement.

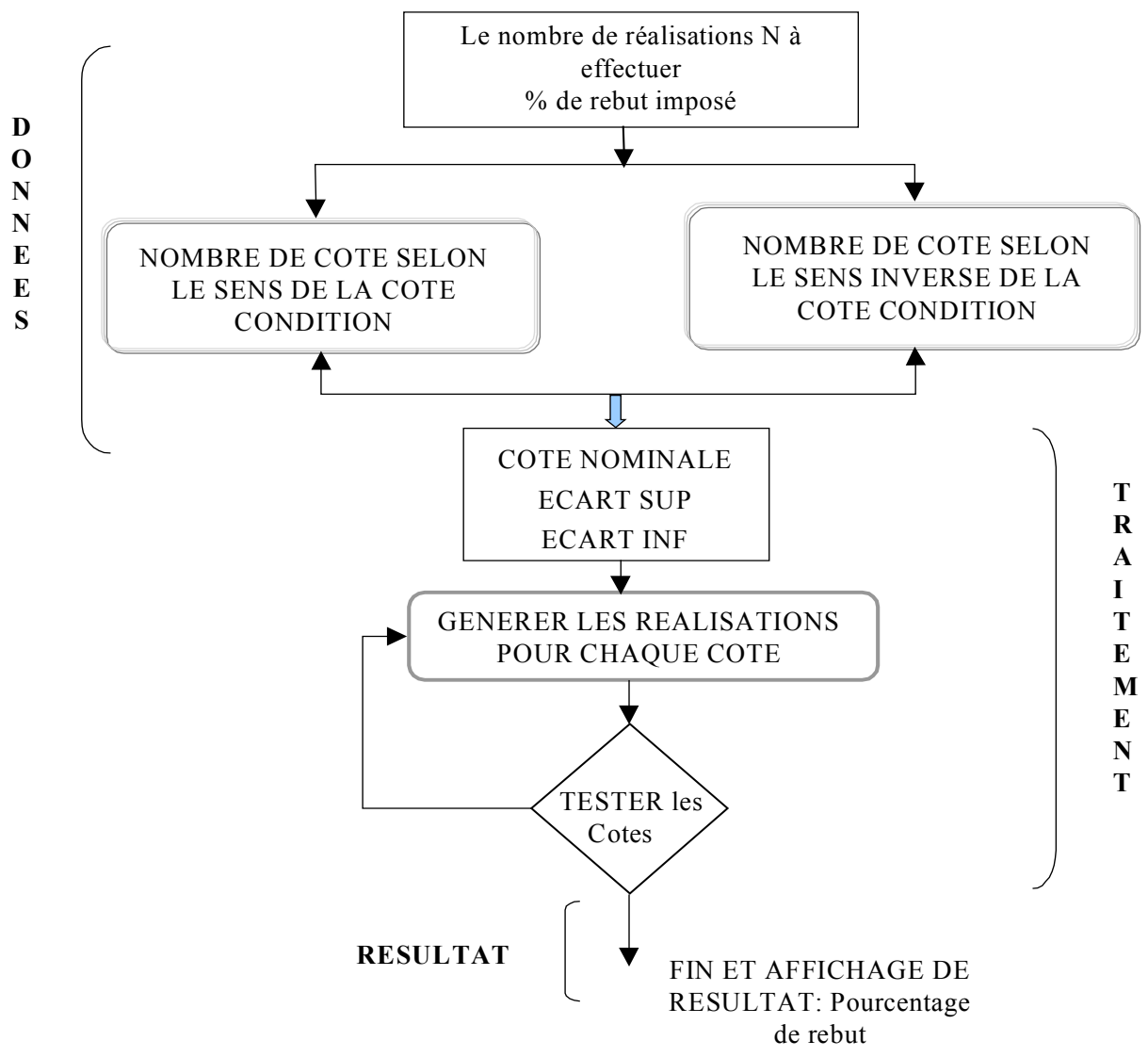

Figure 12 : Algorithme d'application de la MMC

Selon la figure 12, l'application de la MMC consiste à effectuer la génération de variables de manière aléatoire selon une loi normale autour de la moyenne de chaque cote 
de la chaîne de cotes associée à l'assemblage. Il s'agit ensuite de vérifier la conformité de la chaîne à partir de points générés vis-à-vis de la cote condition. Les points générés correspondent à des pièces réalisées en virtuel. Un test sur le nombre des pièces ou d'assemblage ne vérifiant pas la chaîne (pourcentage de rebut) vis-à-vis du pourcentage de rebut imposé par l'utilisateur permet de se décider sur le tolérancement spécifié. Cela passe par une procédure d'optimisation qui consiste à :

i) Déterminer le pourcentage de rebut de pièces ou d'assemblages de la chaîne : pourcentage de rebut calculé.

ii) Comparer le pourcentage de rebut calculé à celui imposé.

iii) Modifier les écarts (intervalles de tolérances) sur la base de la comparaison précédente :

- Si \% rebut calculé $<\%$ rebut imposé $\pm \varepsilon$ alors élargir les intervalles de tolérances les plus fines. ( $\varepsilon$ est l'écart attribué au \% de rebut imposé)

- Si $\%$ rebut calculé $>\%$ rebut imposé $\pm \varepsilon$ alors réduire les intervalles de tolérances les plus larges.

- Sinon, accepter le résultat.

Cette boucle d'optimisation est représentée par la figure suivante ;

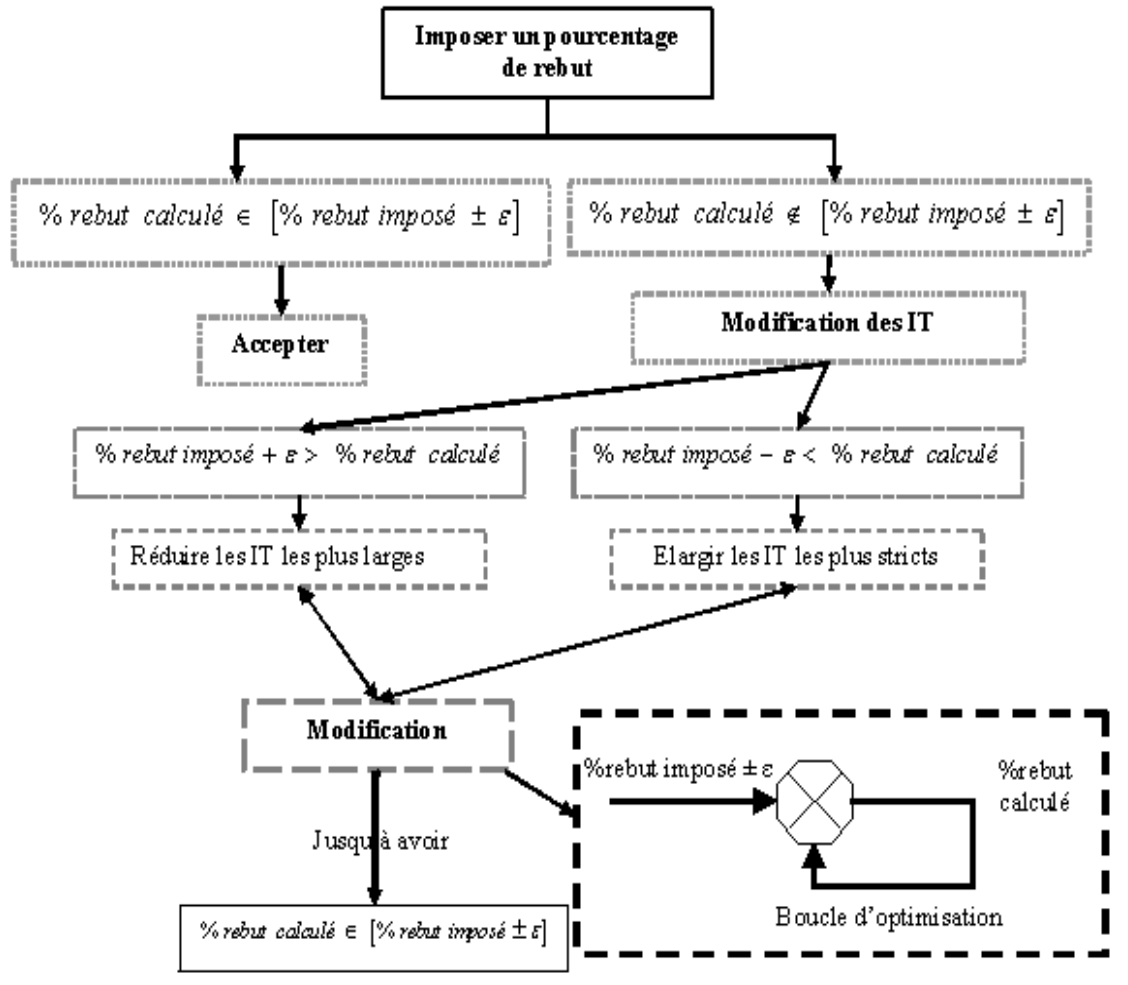

Figure 13 : Algorithme d'optimisation des tolérances en fonction du \% de rebut 


\section{IV.3. Synthèse}

La méthodologie de spécification et de vérification des tolérances proposée se base essentiellement sur deux méthodes qui s'avèrent complémentaires. La MPC, qui a pour objectif soit de spécifier des tolérances à partir d'une cote condition, soit de vérifier un tolérancement déjà définit. La MMC, qui est une méthode statistique, est fondée sur le principe de sacrifier un pourcentage d'échec d'assemblages tout en élargissant des intervalles de tolérances éventuellement déterminés par la MPC. Cette méthode puise ses avantages par la prise en compte des caractéristiques de production (capabilité machine, distribution normale des pièces autour des valeurs nominales) dès la phase de spécification et vérification des tolérances (phase de conception). Ce qui est en parfaite concordance avec les principes de l'ingénierie simultanée.

\section{Mise en ouvre informatique et validation sur un exemple}

\section{V.1. Outil de tolérancement : TOL_ANALYSES}

La maquette informatique est développée sous la plate forme de développement Matlab 7 qui est caractérisée par sa richesse fonctionnelle ainsi que sa rapidité et son efficacité de simulation. La figure 14 représente le menu principal de l'outil de tolérancement proposé :TOL_ANALYSES. A partir de ce menu principal, le concepteur peut choisir d'appliquer une stratégie d'analyse ou de synthèse (optimisation) des valeurs de tolérances. Ce choix lui permet d'accéder à l'une des interfaces de la figure 15. Sur l'une de ces interfaces, il pourra choisir de mettre en œuvre l'une des méthodes : MPC ou MMC.

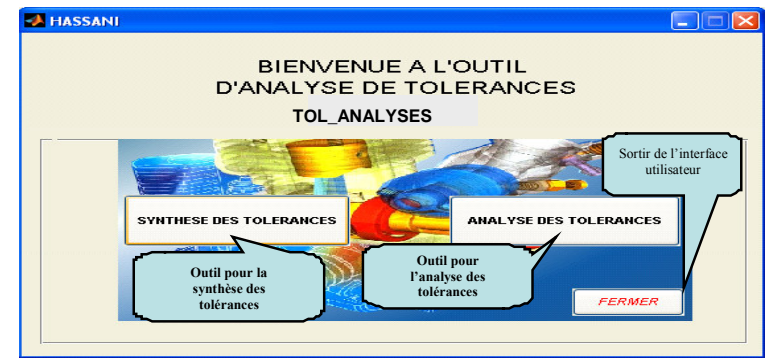

Figure 14: Menu principal de l'outil TOL_ANALYSES 

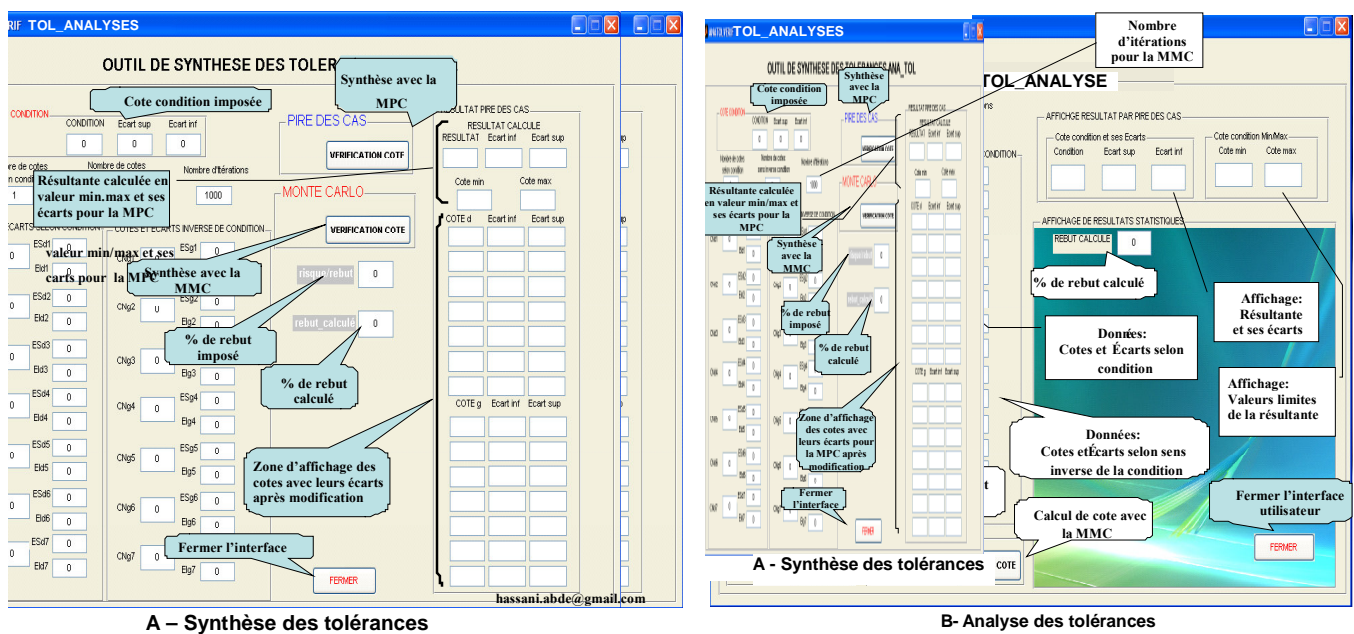

Figure 15: Menu TOL_ANALYSES du choix de la stratégie de tolérancement

\section{V.2. Exemple de validation}

Afin de montrer les principales fonctionnalités de l'outil d'aide au tolérancement TOL ANALYSES, un exemple d'un montage de couvercle d'hélice dans un micro moteur à deux temps [23] est développé. Dans un premier, le concepteur calcule la cote condition en se basant sur la chaîne de cote préalablement spécifiée. Pour cela, il met en œuvre la méthode MPC à partir du menu principal de l'outil. Dans un deuxième temps, le concepteur utilise la méthode MMC en vue d'optimiser les tolérances contributeurs. Pour cela, il doit se fixer un pourcentage de rebut. Celui-ci sera choisi non seulement en concertation avec l'équipe de conception pour l'aspect fonctionnel, mais aussi avec l'équipe des méthodes pour l'aspect coût. L'optimisation des intervalles de tolérances des cotes contributeurs est basée sur l'algorithme de la figure 13. La figure suivante représente à la fois l'exemple ainsi que la chaîne de cotes et les intervalles de tolérances associées.

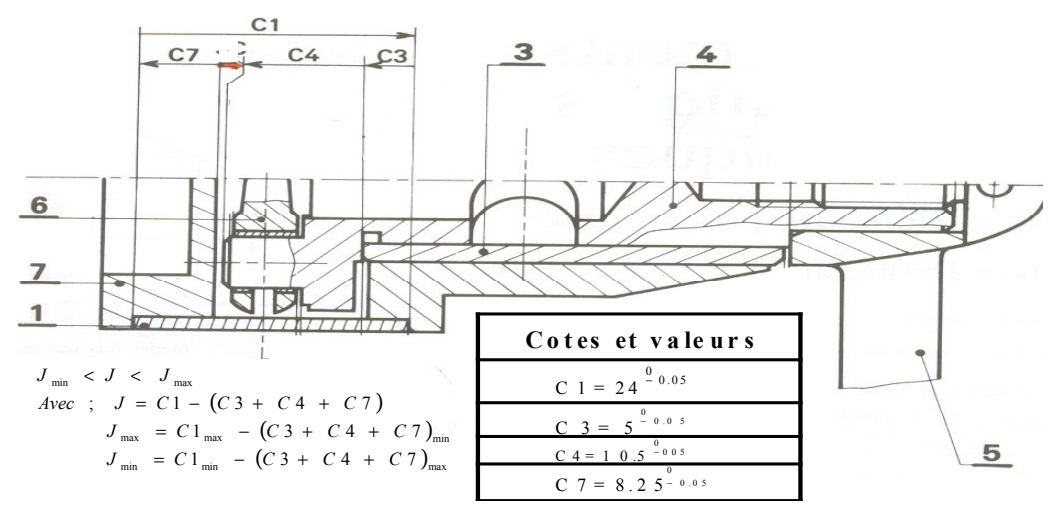

Figure 16 : Exemple de validation 


\section{V.2.1. Application de la méthode MPC}

La mise en œuvre de la méthode MPC permet d'avoir le résultat suivant :

$J \max =O .4$. Cela veut dire que IT $=0.2$. L'observation des cotes contributeurs $J$ min $=0.2$

montre que les IT associées sont serrées. Cela peut poser de sérieux problèmes de fabrication en produisant des pièces non fonctionnelles. Pour remédier à ce problème, le concepteur envisage de relaxer les tolérances serrées tout en sacrifiant un \% de rebut. Les IT associées aux cotes $\mathrm{C} 1$ et $\mathrm{C} 3$ sont considérées parmi les IT serrées (figure 16). Pour se faire, il fait appel à la méthode MMC.

\section{V.2.2. Application de la méthode MMC}

La mise en œuvre de la MMC nécessite la spécification de certaines informations, à savoir le \% de rebut imposé ainsi que le nombre $\mathrm{N}$ de discrétisation des IT. Les histogrammes de la figure suivante représentent les valeurs des cotes de la chaîne simulée.
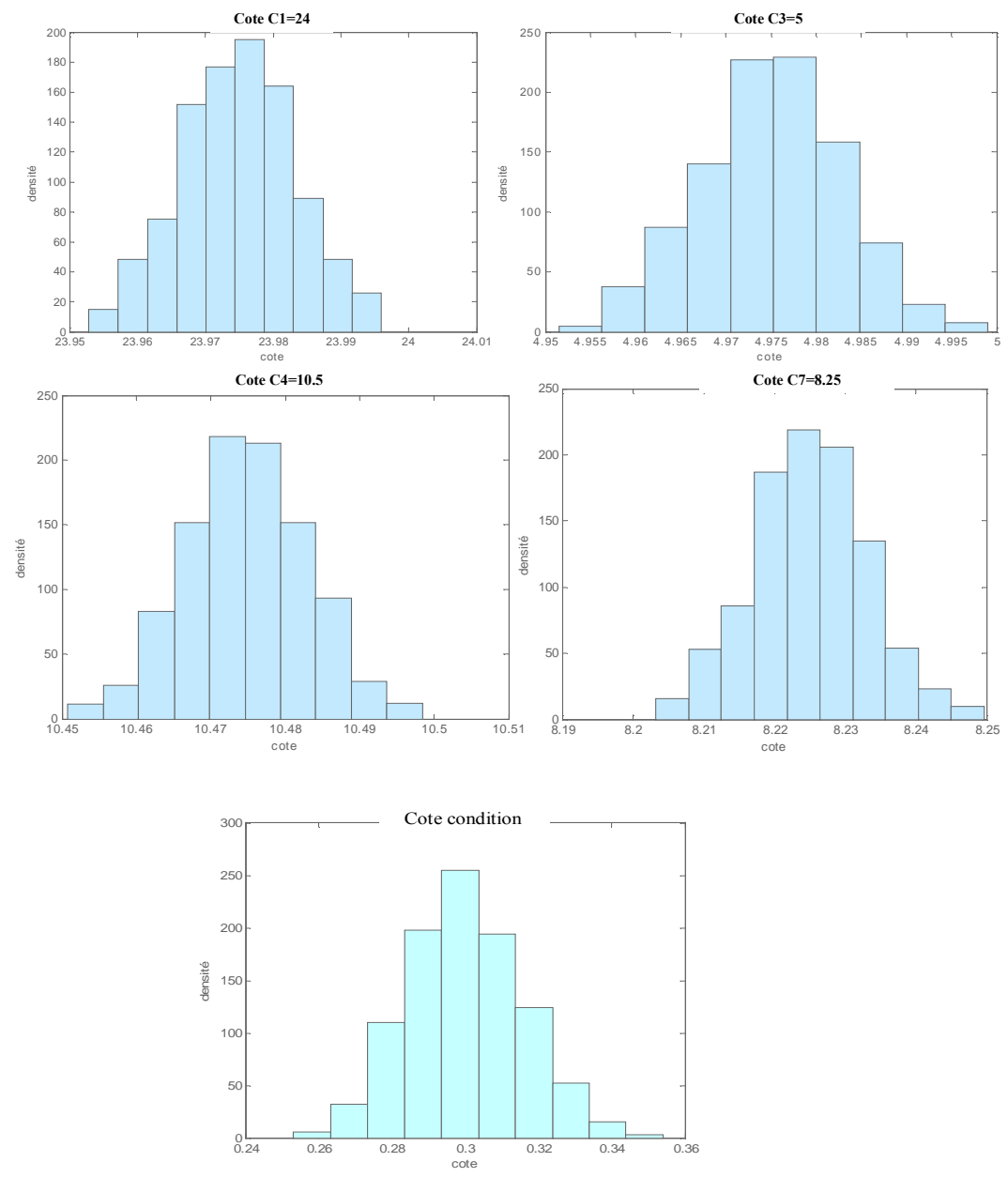

Figure 17 : Histogrammes des cotes générées de la chaîne 
Ces résultats peuvent également être récupérés sous forme de fichier texte en vue de post-traitements. Le tableau de la figure suivante retrace les résultats des histogrammes.

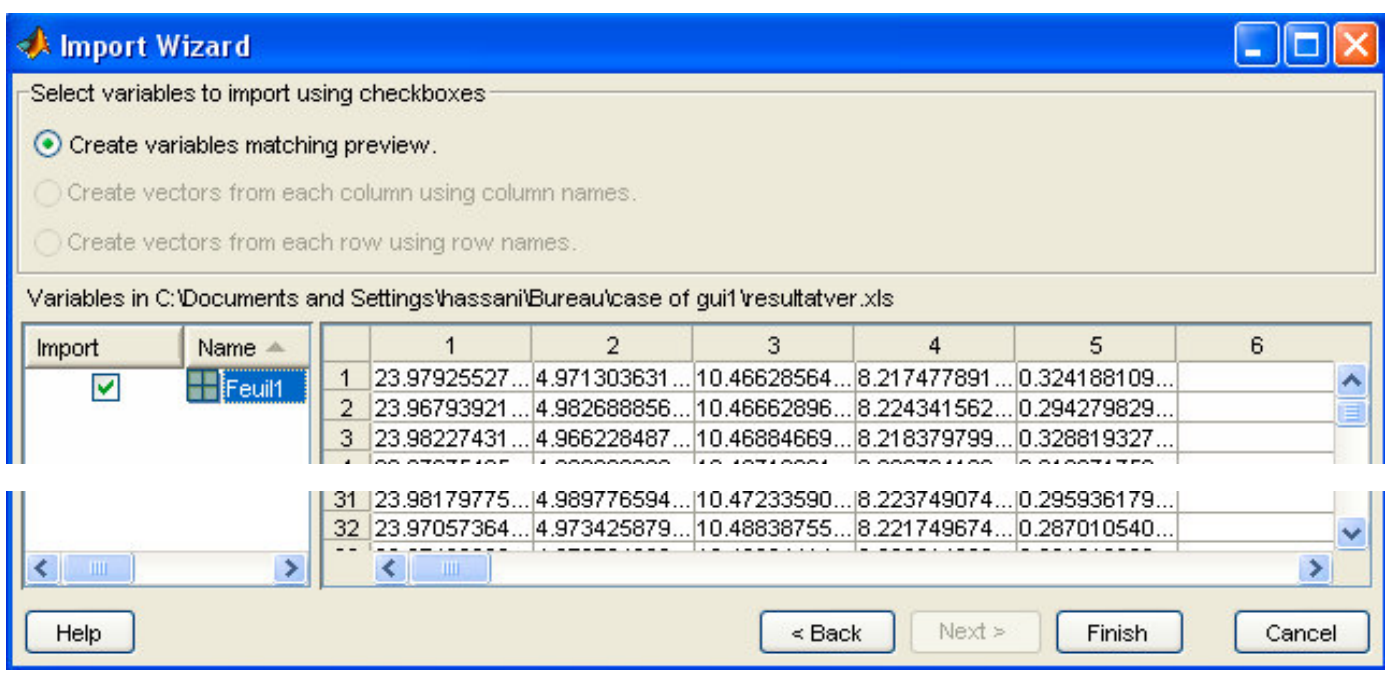

Tableau 3 : Valeurs des cotes estimées

Cette étude est basée sur l'échantillonnage aléatoire de $\mathrm{N}=1000$ itérations pour chaque cote de la chaîne étudiée. Le tableau suivant représente les principales caractéristiques de chaque cote (écart type, \% de rebut, IT arithmétique, IT statistiques, etc.). Il est à constater que le $\%$ de rebut ou de pièces non-conformes est de $0 \%$ pour toutes les pièces qui contribuent à la chaîne de cotes. Cela est dû au fait que la simulation MMC a été faite avec les IT obtenues par la MPC.

\begin{tabular}{|c|c|c|c|c|c|}
\hline Pièce & $\mathbf{C 1}$ & $\mathbf{C 3}$ & $\mathbf{C 4}$ & $\mathbf{C 7}$ & J \\
\hline Ecart type & 0.0083 & 0.0083 & 0.0083 & 0.0083 & $\mathbf{0 . 0 1 6}$ \\
\hline moyenne & 23.975 & 4.975 & 10.475 & 8.225 & $\mathbf{0 . 3}$ \\
\hline \% de rebut calculé & 0. & 0. & 0. & 0. & $\mathbf{0 .}$ \\
\hline Limite sup arithmétique & 24. & 5. & 10.5 & 8.25 & $\mathbf{0 . 4}$ \\
\hline Limite inf arithmétique & 23.95 & 4.95 & 10.45 & 8.20 & $\mathbf{0 . 2}$ \\
\hline Limite inf statistique & $\mathbf{2 3 . 9 5 2 9}$ & $\mathbf{4 . 9 5 1 3}$ & $\mathbf{1 0 . 4 5 0 1}$ & $\mathbf{8 . 2 0 1 1}$ & $\mathbf{0 . 2 5 2 9}$ \\
\hline Limite sup statistique & $\mathbf{2 3 . 9 9 7 7}$ & $\mathbf{4 . 9 9 9 5}$ & $\mathbf{1 0 . 4 9 8 4}$ & $\mathbf{8 . 2 4 6 9}$ & $\mathbf{0 . 3 4 5 4}$ \\
\hline ITar & $\mathbf{0 . 0 5}$ & $\mathbf{0 . 0 5}$ & $\mathbf{0 . 0 5}$ & $\mathbf{0 . 0 5}$ & $\mathbf{0 . 2}$ \\
\hline ITst & $\mathbf{0 . 0 4 4 7}$ & $\mathbf{0 . 0 4 8 2}$ & $\mathbf{0 . 0 4 8 2}$ & $\mathbf{0 . 0 4 5 8}$ & $\mathbf{0 . 0 9 2 5}$ \\
\hline
\end{tabular}

Tableau 4 : Tableau descriptif de la simulation MMC

Statistiquement, les résultats trouvés caractérisent essentiellement :

- Les valeurs moyennes sont arithmétiquement additionnées ;

$$
\begin{aligned}
& \mu_{J}=\mu_{C 1}-\mu_{C 3}-\mu_{C 4}-\mu_{C 7} \\
& \Rightarrow 0.3=23.975-4.975-10.475-8.225
\end{aligned}
$$

- Les valeurs des variances sont quadratiquement additionnées ; 


$$
\begin{aligned}
& \sigma_{j}^{2}=\sigma_{c 1}^{2}-\sigma_{c 3}^{2}-\sigma_{c 4}^{2}-\sigma_{c,}^{2} \\
& \Rightarrow \sigma_{j}^{2}=(0.016)^{2}=(0.0083)^{2}+(0.0083)^{2}+(0.0083)^{2}+(0.0083)^{2}
\end{aligned}
$$

De plus, les intervalles de tolérances statistiques sont comprises dans les intervalles de tolérances arithmétiques: $I T_{s t} \leq I T_{a r}$. Ce constat vient de fait qu'en utilisant l'approche arithmétique, la dispersion maximale des valeurs de l'écart représentée par $\mathrm{IT}_{\mathrm{ar}}$ est supérieure à la très faible probabilité de la dispersion des valeurs représentée par $\mathrm{IT}_{\mathrm{st}}$ statistique. Ce qui montre que les cas extrêmes sont pris en compte dans une approche arithmétique que dans une approche statistique. Cette constatation a été annoncée dans [6]. Elle prodigue au concepteur une idée sur les cotes à modifier et de connaitre celles qui sont plus sensible vis-à-vis à toute modification. Ce qui lui permet de choisir la façon d'intervenir tout en élargissant ou en réduisant les IT convenables. En vue de résoudre les problèmes de fabrication dus aux IT jugées serrées. Le concepteur décide d'élargir les IT associées aux cotes $\mathrm{C} 1$ et $\mathrm{C} 3$ puis refaire la simulation MMC.

\section{V.2.3. Nouvelle simulation MMC}

Lorsque la nouvelle simulation MMC adopte un pourcentage de rebut de $1 \%$, les IT des cotes $\mathrm{C} 1$ et $\mathrm{C} 3$ sont transformés (tableau 5).

\begin{tabular}{|l|c|c|}
\hline & $\mathrm{C} 1$ & $\mathrm{C} 3$ \\
\hline IT initial & 0 & 0 \\
\hline IT après 2 simulations & -0.05 & -0.05 \\
\hline IT après 4 simulations & 0.01 & 0.02 \\
& -0.05 & -0.05 \\
\hline IT final & 0.042 & 0.042 \\
& -0.053 & -0.043 \\
\hline
\end{tabular}

Tableau 5 : Résultats de la simulation avec un rebut de $1 \%$

La simulation MMC, basée sur un échantillonnage aléatoire de $\mathrm{N}=1000$ itérations, aura pour objectif d'optimiser ces IT sur la base du taux de rebut calculé en fonction de celui imposé. Cela est illustré par la figure 18 suivante.

Cadre d'optimisation:

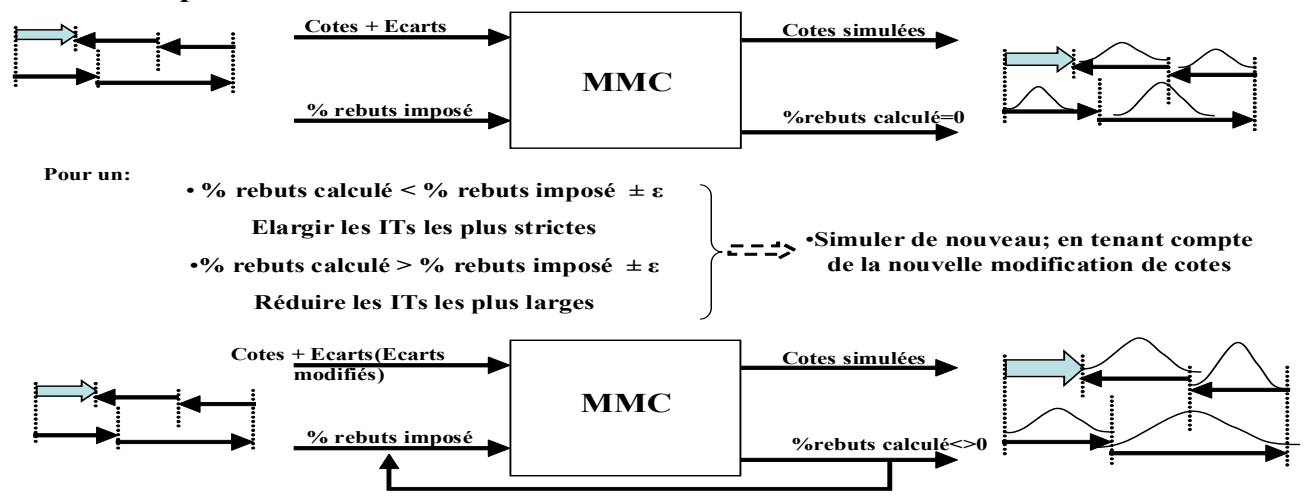

Refaire la simulation tant que le \% rebuts calculé n'est pas dans une gamme donnée(imposée).

Figure 18 : Boucle d'optimisation

La simulation MMC permet enfin d'aboutir aux résultats affichés dans le tableau suivant : 


\begin{tabular}{|c|c|c|c|c|c|}
\hline Pièce & $\mathbf{C 1}$ & $\mathbf{C 3}$ & $\mathbf{C 4}$ & $\mathbf{C 7}$ & $\mathbf{J}$ \\
\hline Ecart type & 0.0095 & 0.0110 & 0.0081 & 0.0077 & $\mathbf{0 . 0 1 8 5}$ \\
\hline Moyenne & 23.979 & 4.984 & 10.475 & 8.225 & $\mathbf{0 . 2 9 4}$ \\
\hline $\begin{array}{c}\text { \% de rebut } \\
\text { calculé }\end{array}$ & $0.28 \%$ & $0.28 \%$ & $0.28 \%$ & $0.28 \%$ & $\mathbf{0 . 2 8 \%}$ \\
\hline $\begin{array}{c}\text { Limite sup } \\
\text { arithmétique }\end{array}$ & 24.01 & 5.02 & 10.50 & 8.25 & $\mathbf{0 . 4 1}$ \\
\hline $\begin{array}{c}\text { Limite inf } \\
\text { arithmétique }\end{array}$ & 23.95 & 4.95 & 10.45 & 8.20 & $\mathbf{0 . 1 8}$ \\
\hline $\begin{array}{c}\text { Limite sup } \\
\text { statistique }\end{array}$ & $\mathbf{2 4 , 0 0 4 9}$ & $\mathbf{5 , 0 1 4 2}$ & $\mathbf{1 0 , 4 9 6 3}$ & $\mathbf{8 , 2 4 5 6}$ & $\mathbf{0 , 3 5 1 7}$ \\
\hline $\begin{array}{c}\text { Limite inf } \\
\text { statistique }\end{array}$ & $\mathbf{2 3 , 9 5 4 7}$ & $\mathbf{4 , 9 5 5 0}$ & $\mathbf{1 0 , 4 5 3 6}$ & $\mathbf{8 , 2 0 4 0}$ & $\mathbf{0 , 2 4 0 0}$ \\
\hline ITar & $\mathbf{0 . 0 6}$ & $\mathbf{0 . 0 7}$ & $\mathbf{0 . 0 5}$ & $\mathbf{0 . 0 5}$ & $\mathbf{0 . 2 3}$ \\
\hline ITst & $\mathbf{0 , 0 5}$ & $\mathbf{0 , 0 5 9 2}$ & $\mathbf{0 , 0 4 2 7}$ & $\mathbf{0 , 0 4 1 6}$ & $\mathbf{0 , 1 1 1 6}$ \\
\hline
\end{tabular}

Tableau 6 : Tableau récapitulatif de la simulation MMC

La boucle d'optimisation des IT des cotes contributrices permet d'aboutir à un tolérancement optimisé sur la base de la capabilité des machines de production. Moyennant cette méthodologie d'aide à la spécification et à la vérification des tolérances, le concepteur est désormais capable de prendre en compte, dès la conception, les aspects liés à la production. Cela correspond bien aux principes de l'ingénierie simultanée.

\section{Conclusion}

Dans ce papier une méthodologie d'analyse est de synthèse des tolérances a été proposée. L'analyse des tolérances consiste à vérifier à postériorité la réalisation des conditions fonctionnelles préalablement spécifiées à partir du tolérancement instancié de toutes les pièces du mécanisme. Tandis que la synthèse des tolérances consiste, à partir des spécifications exprimant les conditions fonctionnelles entre pièces, à générer un tolérancement des différentes pièces du mécanisme étudié respectant ces conditions. Cette méthodologie intègre deux principales méthodes. La méthode de Monte Carlo (MMC) qui est une méthode statistique est fondée sur le principe de sacrifier un pourcentage d'échec d'assemblages tout en augmentant ou diminuant les intervalles de tolérances éventuellement synthétisées par la méthode au pire des cas (MPC). Cette méthode s'avère intéressante puisqu'elle permet la prise en compte des caractéristiques de production (capabilité machine, distribution normale des pièces) dès la phase de conception.

Un algorithme d'optimisation des tolérances permet au concepteur de mieux répartir la cote condition sur les intervalles de tolérances contributeurs à la chaîne de cotes sur la base du \% de rebut calculé en fonction du \% de rebut imposé. Le concepteur peut ainsi élargir les intervalles de tolérances les plus serrées et/ou réduire les intervalles de tolérances les plus larges. Cela dépend principalement de la capabilité des machines de production.

La méthodologie proposée confère au concepteur de ne plus raisonner en terme d'états limites des tolérances mais d'une distribution plus réaliste (la production ne produit jamais des états limites des cotes) et de prendre en compte le contexte de 
production (capabilité machine, distribution normale). Ce qui est en concordance avec les principes de l'ingénierie simultanée.

La méthodologie proposée se limite au tolérancement dimensionnel linéaire. Une aide efficace à la démarche de spécification et de vérification des tolérances nécessite la prise en compte d'autres types de tolérancement, en l'occurrence, le tolérancement géométrique (forme, position, orientation, battement). Elle suppose que les pièces sont considérées comme des solides rigides. Il est important de prendre en compte l'aspect déformation des pièces (due aux conditions de montage et de fonctionnement...) lors de la spécification et de la vérification des tolérances.

\section{Références bibliographiques}

[1] E. Pairel, M. Giordano, «Le tolérancement dimensionnel Norme; ISO 8015:1985, Formation au tolérancement ISO : Demi-journée de synthèse », supports de cours, SYMME, Université de Savoie, 2000

[2] P. Bourdet «Spécification géométrique des produits (GPS) », polycopiés de cours, 2005, http://www.lurpa.ens-cachan.fr/ bourdet/

[3] A. Etienne, «Intégration Produit / Process par les concepts d'activités et de caractéristiques clés - Application à l'optimisation de l'allocation des tolérances géométriques », Thèse de Doctorat, Université Paul Verlaine, Octobre 2007.

[4] L. Markvoort, «Prise en compte de la nature flexible des matériaux pour l'analyse de tolérances ", actes du 9ième colloque National AIP PPRIMECA, Université de Valenciennes, avril 2005.

[5] L. Markvoort, D. Deneux, «Un état de l'art sur les approches d'analyse de tolérances », Séminaire national AIP PRIMECA sur le tolérancement, ENS de Cachan, France, septembre 2006.

[6] L. Markvoort, «Méthodologie d'analyse statistique de tolérances dans les assemblages impliquant des composant déformables », Université de Valenciennes, Thèse de Doctorat, juin 2007.

[7] M. Temmerman, "Analyse et synthèse 'au pire des cas' et statistique dans l'environnement CFAO, LISMMA-ISMCM, Ecole centrale de Paris, Thèse de Doctorat. 2001.

[8] M. Pillet, Duret D., Sergent S. "Weighted Inertial Tolerancing", Quality Engineering, Vol. 17-2005, N4, pp 667-693.

[9] M. Pillet Maurice Pillet, P.A. Adragna, D. Denimal, «Monographie sur le tolérancement inertiel », Laboratoire SYMME, Université de Savoie, France, pp.1-71, année 2007. http://web.qlio.univ-savoie.fr/pillet/

[10] M. Pillet, J Breton, «Le tolérancement Inertiel $\mathrm{Ou}$ comment faire $\mathrm{du}$ tolérancement statistique en garantissant la fonction du produit », CAD Magazine $\mathrm{N}^{\circ} 133$ août - septembre 2006 pp 56-59. 
[11] M. Bouix, M. Pillet, «Comment garantir la fonctionnalité d'un assemblage en travaillant autrement avec les fournisseurs? Les apports du tolérancement inertiel », $2^{\text {ième }}$ Journée Européenne du Tolérancement, JET 2006, Annecy, France, Juin 2006. http://www.jet2006.fr

[12] S. Bhide, J. K. Davidson, J. J. Shah, "A new mathematical model for geometric tolerances as applied to axes", CD-ROM Proceedings, ASME Design Engr. Technical Conf's. (29th Design Automation Conf.), Chicago, IL, Sept. 2-6, 2003, Paper \#DETC2003/DAC-48736, 9.

[13] P. A. Adragna Tolérancement des Systèmes Assemblés, une approche par le Tolérancement Inertiel et Modal, Thèse de Doctorat, Université de Savoie décembre 2007.

[14] B. Anselmetti, M. Radouani, « Calcul statistique des chaînes de cotes avec des distributions hétérogènes non indépendantes » Conception et Production Intégrées : CPI'2003. Meknès 22, 23 \& 24 octobre 2003, pages 1 à 16, 2003.

[15] J. C. Breart, Identifier et traiter les risques produit - process en relation avec les spécifications fonctionnelles, $2^{\text {ième }}$ Journée Européenne du Tolérancement, JET 2006, Annecy, France, Juin 2006. http://www.jet2006.fr

[16] F. Germain, «Tolérancement Statistique Tridimensionnel », Intégration en CFAO, Thèse de Doctorat, Université de Savoie, Annecy, Octobre 2007.

[17] M.N. Islam, "Functional dimensioning and tolerancing software for concurrent engineering applications", Computers in Industry 54(2): 169-190, 2004.

[18] R. Benea, «Contribution a l'analyse tridimensionnelle de tolérances en design et fabrication », mémoire 121 p, Ecole Polytechnique de Montréal, Canada, 2001.

[19] A. Etienne, «Allocation des spécifications géométriques - Evaluation des coûts impactés par les spécifications géométriques », GDR MACS - GT IS3C, 10 Mars 2006.

[20] B. Anselmetti, S. Bisson, «Emploi des indicateurs d'aptitude pour le traitement statistique d'une chaîne de cotes », Congrès Qualita 2005, CDRom paper, pp. 9198, mars 2005

[21] R. Vandromme, «La capabilité du processus », Technonologies vol. 140, pp 2835 Décembre 2005.

[22] G. Casanova, "Gestion De La Qualité - Techniques Statistiques », support de cours, Institut d'Administration des Entreprises Université Nancy, 22001.

[23] A. Chevalier, « Guide du dessinateur industriel », Edition Hachette 1995. 